\title{
Altered Bicarbonate Reabsorption in the Pancreas of Reserpine-Treated Rabbits-a Model for Cystic Fibrosis
}

\author{
MITCHELL L. SHIFFMAN, ${ }^{(23)}$ ROGER E. SPITZER, PHILLIP T. SWENDER, ${ }^{(25)}$ AND \\ WILLIAM R. GALEY \\ Robert C. Schwartz Cystic Fibrosis Center, Department of Pediatrics State University of New York, Upstate Medical \\ Center, Syracuse, New York and Department of Physiology, University of New Mexico School of Medicine, \\ Albuquerque, New Mexico, USA
}

\begin{abstract}
Summary
Pancreatic juice was collected from rabbits treated for 7 days with reserpine as well as from untreated controls. In treated rabbits, the volume of pancreatic juice secreted $(0.94 \pm 0.31 \mathrm{mg})$ and the maximum pancreatic juice flow rates $(66.1 \pm 7.1 \mathrm{mg} /$ $(\mathrm{min} \cdot \mathrm{g})$ ffdpw) were both significantly less $(\mathrm{p}<0.005)$ than observed in control animals $(1.60 \pm 0.24 \mathrm{mg}$ and $98.3 \pm 15 \mathrm{mg} /(\mathrm{min} \bullet$ g)ffdpw). The bicarbonate concentration of the juice obtained from reserpinized rabbits was elevated at all flow rates when compared to the juice obtained from controls. When saliva obtained from patients with $C F$ was retroperfused into the pancreatic ductal system of a control rabbit, the resultant pancreatic juice contained an elevated bicarbonate concentration at every given flow rate. In addition, $1 \mathrm{~h}$ after retroperfusion, bicarbonate output was elevated by $15 \%$ despite a reduction in pancreatic juice volume as compared to unretroperfused controls. When pancreatic juice obtained from reserpine-treated rabbits was retroperfused into the ductal system of a control rabbit's pancreas, an elevation in the bicarbonate concentration also resulted. In addition, $1 \mathrm{~h}$ after retroperfusion with reserpinized pancreatic juice, the bicarbonate output increased by $18 \%$ whereas the volume of pancreatic juice secreted during this period of time remained unchanged as compared to controls. No alteration in pancreatic bicarbonate, pancreatic juice volume, or flow rate was observed after retroperfusion with either saline, saliva obtained from control patients or pancreatic juice obtained from control rabbits. The pancreatic juice secreted by reserpinized rabbits, therefore, appeared to contain some type of factor that altered the reabsorption of bicarbonate. This pancreatic factor also appeared to be positively charged because its transport inhibitory properties were lost when the reserpinized juice was mixed with the polyanion heparin $(500 \mathrm{U} / \mathrm{ml})$ before retroperfusion.

Despite these observations, the pancreatic bicarbonate output of treated animals was significantly less than that of controls both $30 \mathrm{~min}(P<0.005)$ and $60 \mathrm{~min}(P<0.010)$ after secretin stimulation. It is believed that this finding is secondary to a $41 \%$ reduction in the volume of pancreatic juice secreted by treated animals. Because the reserpine-treated rabbit has been proposed as a valid model for studying CF, this study proposes that a similar transport inhibitory factor might also be present in the pancreatic secretions of patients with this disease.
\end{abstract}

\section{Abbreviations}

CF, cystic fibrosis

ffdpw, fat free dried pancreatic weight

One of the distinguishing features of patients with $\mathrm{CF}$ is that the secretions produced by their sweat and salivary glands contain elevated concentrations of sodium (20). It is now well established that this abnormality is due to the presence of a sodium transport inhibitory factor, which acts within the ductal lumen of both of these glands by preventing the reabsorption of sodium $(6,7,8)$. The pancreas is also profoundly affected by this disease. Patients with CF secrete a pancreatic juice that contains an increased protein concentration and decreases in the total juice output and bicarbonate output $(2,20,21)$. This organ, however, is not easily accessable within the patient. As a result, very few studies have been performed and it is not currently known whether or not ionic movement within this gland is also perturbed by CF, like it is known to be in the sweat and salivary glands. For this reason, the reserpine-treated animal model of $\mathrm{CF}$, developed and characterized first by Martinez and his collaborators $(3,11,12,14)$ would seem to be extremely useful. Both salivary $(11,12)$ and pancreatic (14) secretions in this animal model closely resemble the secretions produced by patients with $\mathrm{CF}$. It has also been demonstrated that the elevated salivary sodium observed in reserpinized rats is due to a transport inhibitory factor with similar properties to the factor found in both the salivary and sweat glands of patients with $\mathrm{CF}$ (13). More recently, the pancreatic juice of reserpine-treated rabbits has been studied and shown to resemble the pancreatic secretions produced by patients with $\mathrm{CF}$ as well $(16,17)$. This paper represents a continuation of these studies and demonstrates that in addition to low flow rates and a reduced bicarbonate output, the pancreatic juice of reserpinized rabbits also contains a transport inhibitory factor which perturbs the reabsorption of bicarbonate across the ductal system of this gland.

\section{MATERIALS AND METHODS}

Animals. Male New Zealand white rabbits weighing $1.0-1.5 \mathrm{~kg}$ were utilized. Reserpinized animals received seven daily intramuscular injections at a dose of $0.5 \mathrm{mg} / \mathrm{kg}$ of body weight. After a 24-h fast on the seventh day of treatment, each animal was anesthetized with $1.0 \mathrm{ml} / \mathrm{kg}$ of Dial-Urethane (Ciba Pharmaceutical Co., Summit, NJ), a tracheostomy was performed and the femoral artery and vein were cannulated (17). An incision was made in the animal's abdomen and the main pancreatic duct was identified and cannulated. This basic preparation was utilized for all the experiments which follow.

Collection of samples to be retroperfused. Saliva was collected from seven patients with CF and from 5 age- and sex-matched control individuals according to the method of Mangos (7). The ages of the saliva donors ranged from 13-25 years. CF patients donated saliva during routine clinic appointments. The degree of pulmonary and pancreatic involvement varied widely among the donors. Control donors were hospital personnel or patients making routine appointments at the SUNY, Upstate Medical Center 
Pediatric Outpatient Clinic. Each had no significant medical problems.

Immediately after collection, each salivary sample was centrifuged at $3000 \times g$ to remove any particulate matter. The supernatant was then diluted 1:2 with saline stained with $1 / 2 \%$ lissamine green (Sigma Chemical Co., St. Louis, MO). Each salivary sample was then retroperfused into the pancreatic duct of a control rabbit as described below.

Retroperfusion was also performed with pancreatic juice that had been collected earlier in the day from reserpine-treated rabbits and/or from untreated controls. In these experiments the control and reserpinized animals were prepared as described above. After administration of an IV bolus of pure natural secretin $(2 \mathrm{U} / \mathrm{kg})$ (Karolinska Institute, Stockholm, Sweden), pancreatic juice was collected in a polyethylene test tube. The juice was centrifuged at $3000 \times g$ and the supernatant diluted $1: 2$ with $1 / 2 \%$ lissamine green.

Four other test samples were also retroperfused. Pancreatic juice, which was collected from reserpine-treated rabbits earlier that day, was first centrifuged and then diluted 1:2 with a solution containing the polyanion heparin (Sigma Chemical Co., St. Louis, MO) in $1 / 2 \%$ lissamine green. The final heparin activity was 500 $\mathrm{U} / \mathrm{ml}$ before retroperfusion. Poly-L-lysine $\mathrm{HBr}$ of medium chain length, molecular weight 400,000, (Sigma Chemical Co., St. Louis, MO) was dissolved in $1 / 2 \%$ lissamine green to produce a $10^{-6} \mathbf{M}$ solution and utilized. Retroperfusion was also carried out with stained saline and heparin $(500 \mathrm{U} / \mathrm{ml})$ in stained saline, which were utilized as controls.

Experimental procedure. In the first set of experiments, $2 \mathrm{U} / \mathrm{kg}$ of pure natural secretin was administered as an IV bolus via the femoral vein cannula to either control or treated animals. The flow of pancreatic juice began immediately, reached a maximum in 3-5 min and then gradually declined with time during the remaining 50-60 min of the experiment. During this time, pancreatic juice samples were collected at timed intervals under waterequilibrated parafin oil in tared tubes. At the end of each collection period, the tubes were reweighed to obtain a gravimetric determination of the amount of juice produced.

All of the remaining experiments utilized the technique of retroperfusion in control nontreated rabbits. One of the various test samples was drawn into a 3-cc polyethylene syringe and then perfused in a retrograde fashion via the main duct cannula into the pancreatic ductal system of each test animal (Fig. 1). The fluid was held within the ductal system for $2 \mathrm{~min}$ at which time the syringe was removed. An IV bolus of secretin $(2 \mathrm{U} / \mathrm{kg})$ was then administered via the femoral vein cannula. The flow of pancreatic juice began immediately, reached a maximum within 3-5 min and gradually declined with time. Pancreatic juice collected during the first 5 min was discarded. All subsequent pancreatic juice samples were collected at timed intervals under water-equilibrated parafin oil in tared tubes. At the end of each collection period, the tubes

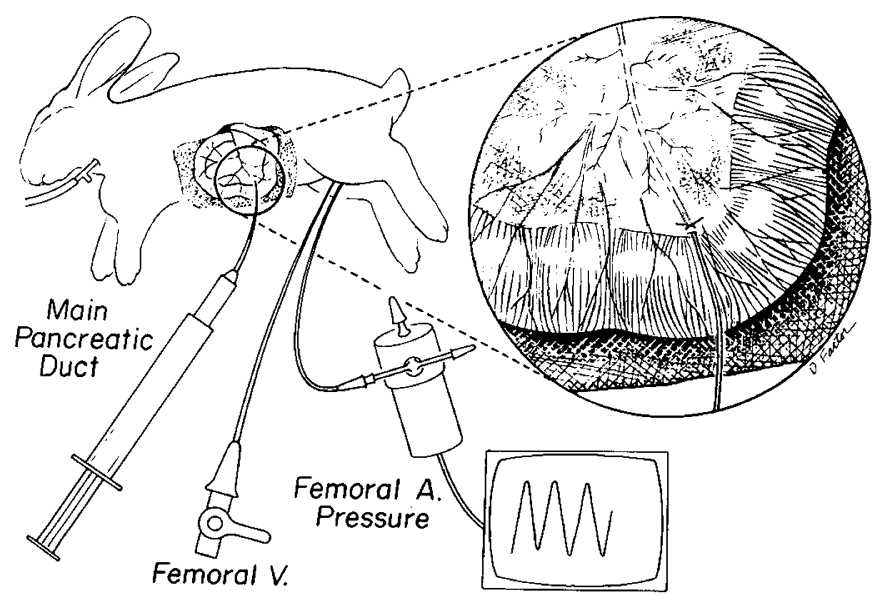

Fig. 1. Schematic illustration of the experimental procedure and the technique of retroperfusion. were again weighed and a gravimetric determination of the juice was obtained.

A $1 / 2 \%$ solution of the biologic dye lissamine green was added to every sample before retroperfusion as stated above. This was done so that the pancreatic ductal system could be visualized during this procedure. By diluting the lissamine green dye with the samples, however, it was sometimes difficult to visualize the perfusate within the ductal system. As a result, in some of the earliest experiments the gland was damaged by "over perfusion" and it failed to fully respond to secretin stimulation. Glandular damage was assumed if the maximum flow rate after secretin stimulation was less than $80 \mathrm{mg} /(\mathrm{min} \cdot \mathrm{g}) \mathrm{ffdpw}$. The results of these experiments were not included in the final data analysis. It was determined that the optimum amount of fluid to retroperfuse should be $0.7-1.0 \mathrm{ml}$. This amount was utilized in all subsequent experiments.

Analytical procedures. Immediately after the collection of each pancreatic juice sample, the $\mathrm{pH}$ and $\mathrm{PCO}_{2}$ were measured by injecting the juice sample into the common chamber of a Corning Model 6A micro blood gas analyzer. At least $75 \mu$ l of juice was required for this determination. The bicarbonate concentration was then calculated from the Henderson-Hasselbalch equation.

At the conclusion of each experiment a solution of $1 \%$ lissamine green was injected into the pancreatic ductal system via the main duct cannula. This enabled the entire gland to be easily identified. The stained pancreas was then carefully dissected free, placed in a test tube and dried in a warming oven. The dried pancreas was then crushed and extracted 5 times with $5 \mathrm{ml}$ of petroleum ether (J.T. Baker Chemical Co., Phillipsburg, NJ). The fat extracted pancreas was redried to a constant weight. Flow rates of pancreatic juice were expressed in terms of $\mathrm{mg}$ of juice/(min.g) of fat free dried pancreatic weight $(\mathrm{mg} / \mathrm{min} \cdot \mathrm{gm}$ ffdpw).

The data points for each set of experiments were analyzed with a Hewlett Packard Model 9800 microprocessor. The best equation fit for each set of data points was calculated utilizing a least squares analysis program. A Student's $t$ test was utilized to determine if the difference between two sample means was significant. A $P$ value of less than 0.05 was considered significant.

\section{RESULTS}

Relationship of bicarbonate concentration to pancreatic flow rates. Figure 2 presents the bicarbonate concentration of the pancreatic juice as a function of the various juice flow rates attained during the course of the experiment. Three observations are readily apparent. The first is that the maximum pancreatic juice flow rates attained by the reserpine-treated rabbits were significantly less ( $P$ $<0.005$ ) than those achieved by the controls. This feature is also described in Table 1. Second, at low flow rates, treated rabbits were found to produce pancreatic juice that contained a bicarbonate concentration approximately $20-40 \mathrm{meq} /$ liter greater than the juice secreted by the controls. Third, as the flow rate increased, the concentration of bicarbonate in the juice approached the same maximal level for both groups of animals, approximately 105 meq/liter. This maximum bicarbonate concentration was achieved in the treated animals despite the initial observation that the maximum flow rate attained by the reserpinized rabbits did not surpass $80 \mathrm{mg} /(\mathrm{min} \cdot \mathrm{g})$ ffdpw; thus, the pancreatic bicarbonate curve of treated animals can be described as shifted to the left.

Effect of retroperfusion on the pancreatic juice flow rate and output. Table 1 demonstrates the effect of retroperfusion on the pancreatic juice flow rate and output. The maximum pancreatic juice flow rates attained after retroperfusion were not significantly different than the maximum flow rates observed in the unperfused control animals. In addition, retroperfusion did not alter the volume of juice secreted in $1 \mathrm{~h}$ except in one instance where the juice output after retroperfusion with $C F$ saliva was noted to be reduced. On the other hand, both the maximum flow rate and the volume of pancreatic juice secreted by reserpinized animals were significantly reduced $(P<0.005)$ when compared to controls.

Effect of retroperfusion on the bicarbonate concentration. The 


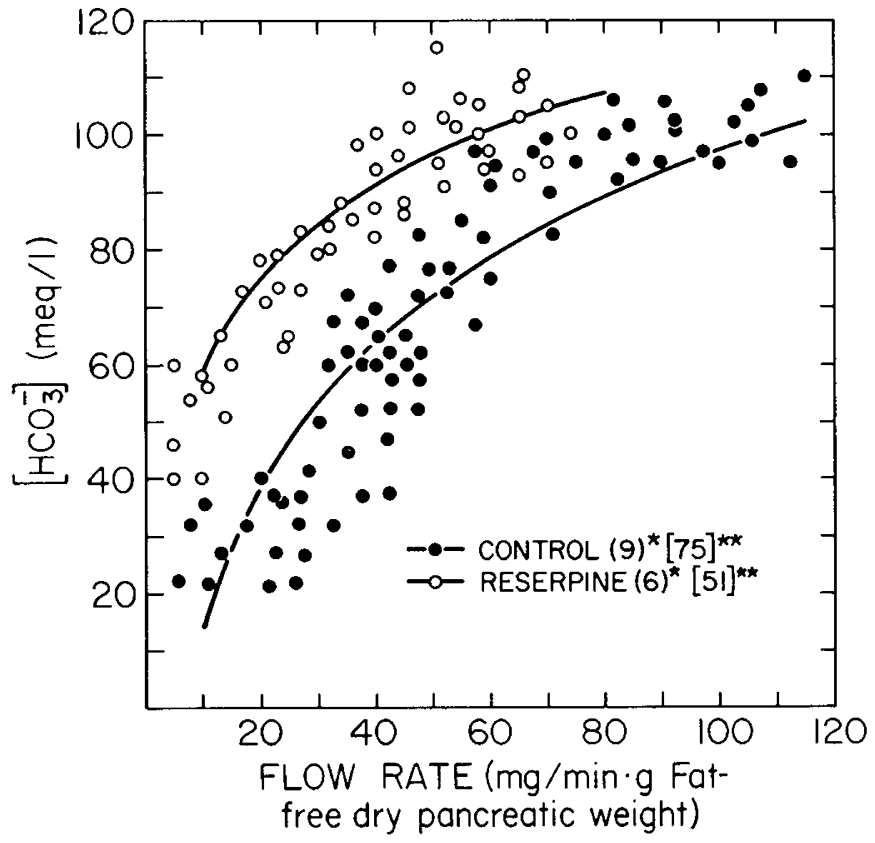

Fig. 2. Bicarbonate concentration as a function of the pancreatic juice flow rate. Each point represents a single pancreatic juice sample. The solid lines represent the best equation fit for each set of data points. * Number in parentheses represents the number of animals used in the study. ** Number in brackets represents the total number of sample determinations.

Table 1. Maximum pancreatic juice flow rates and bicarbonate outputs $1 \mathrm{~h}$ after secretin stimulation (values are given as \pm S.E.M.)

\begin{tabular}{lcc}
\hline & $\begin{array}{c}\text { Flow rate } \\
(\mathrm{mg} /(\mathrm{min} \cdot \mathrm{g}) \\
\mathrm{ffdpw})\end{array}$ & $\begin{array}{c}\text { Output } \\
(\mathrm{mg})\end{array}$ \\
\hline Control & $98.3 \pm 12.5(6)$ & $1.60 \pm 0.24(6)$ \\
Reserpine treated & $66.1 \pm 07.1(5)^{1}$ & $.94 \pm 0.31(5)^{1}$ \\
$\quad \begin{array}{l}\text { After retroperfusion with: } \\
\quad \text { CF saliva }\end{array}$ & $98.7 \pm 13.9(6)$ & $1.26 \pm 0.20(6)^{2}$ \\
$\quad \begin{array}{l}\text { Control saliva } \\
\text { Pancreatic juice from re- } \\
\quad \text { serpinized animals }\end{array}$ & $105.9 \pm 09.4(5)$ & $1.50 \pm 0.37(5)$ \\
$\quad \begin{array}{l}\text { Pancreatic juice from con- } \\
\text { trol animals }\end{array}$ & $110.4 \pm 07.9(4)$ & $1.32 \pm 0.28(4)$ \\
$\quad \begin{array}{l}\text { Pancreatic juice from re- } \\
\quad \text { serpinized animals plus }\end{array}$ & $100.6 \pm 04.1(5)$ & $1.43 \pm 0.24(4)$ \\
$\quad$ heparin & & \\
$\quad$ Poly-L-lysine & & $1.49 \pm 0.26(5)$ \\
\hline
\end{tabular}

${ }^{1}$ Significantly different from control; $P<0.005$.

${ }^{2}$ Significantly different from control; $P<0.025$.

effect that retroperfusion has upon the pancreatic bicarbonate concentration is illustrated in Figures 2-6. Retroperfusing with saliva obtained from either control individuals or from patients with $\mathrm{CF}$ is presented in Figure 3. This demonstrates that CF saliva elevated the bicarbonate concentration of the resultant pancreatic juice by approximately $40 \mathrm{meq} / \mathrm{liter}$ at all but the highest flow rates where the bicarbonate concentration approximated that of controls (105 meq/liter). By contrast, retroperfusion with saliva obtained from age- and sex-matched control individuals did not alter the normal pancreatic bicarbonate relationship.

When pancreatic juice collected from reserpine-treated rabbits was retroperfused into the ductal system of control non-treated rabbits, the resultant pancreatic juice contained an elevated bicarbonate concentration at every given flow rate (Fig. 4). At low flow rates, this elevation approximated $40 \mathrm{meq} / \mathrm{liter}$. At high flow rates,

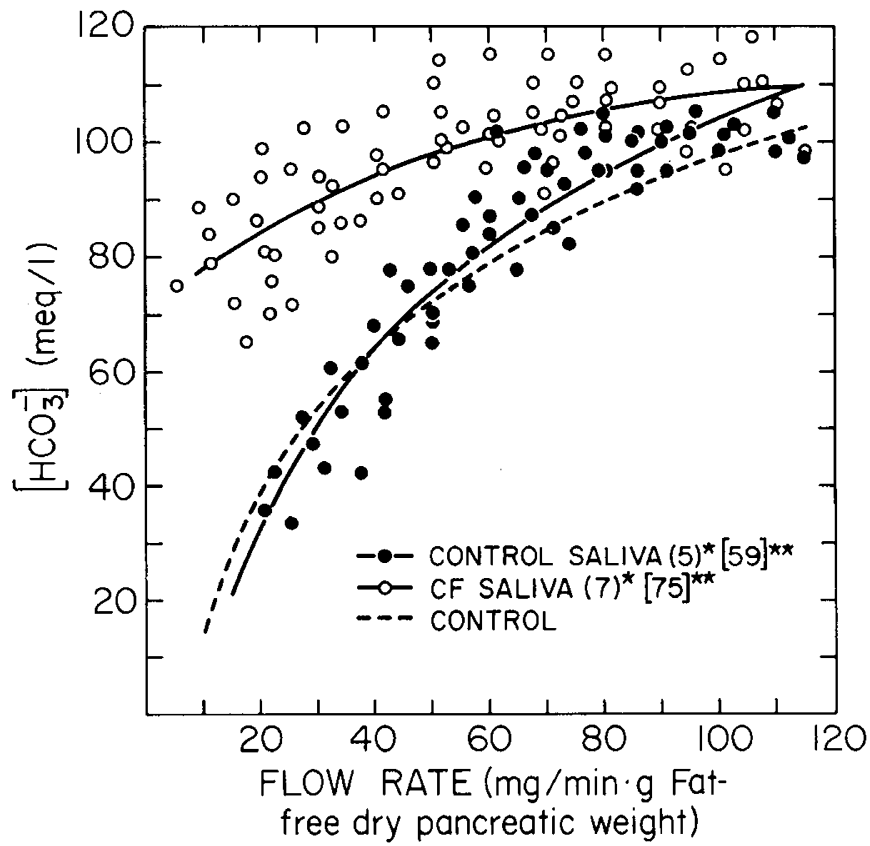

Fig. 3. Bicarbonate concentration as a function of the pancreatic juice flow rate after retroperfusion with saliva obtained from control or $\mathrm{CF}$ patients. Each point represents a single pancreatic juice sample. The solid lines represent the best equation fit for each set of data points. The dotted line is the control curve from Figure 2. ${ }^{*}$ Number in parentheses represents the number of animals used in the study. ${ }^{* *}$ Number in brackets represents the total number of sample determinations.

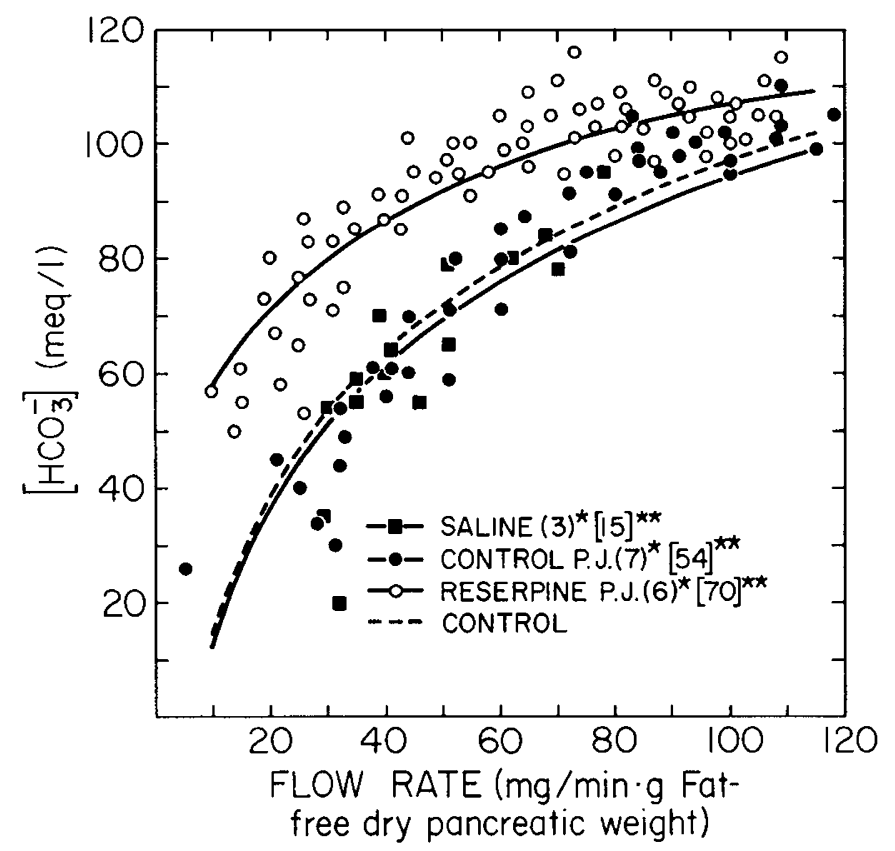

Fig. 4. Bicarbonate concentration as a function of the pancreatic juice flow rate after retroperfusion with saline or pancreatic juice obtained from control or reserpine-treated rabbits. Each point represents a single pancreatic juice sample. The solid lines represent the best equation fit for each set of data points. The dotted line is the control curve from Figure 2. * Number in parentheses represents the number of animals used in the study. ${ }^{* *}$ Number in brackets represents the total number of sample determinations.

however, the elevation in pancreatic bicarbonate became less significant, approximating only $5 \mathrm{meq} / \mathrm{liter}$. No substantial alteration in the normal pancreatic bicarbonate curve was observed after retroperfusion with juice obtained from control rabbits or 
after retroperfusion with saline. On the other hand, when the pancreatic juice obtained from reserpinized animals was mixed with the poly-anion heparin $(500 \mathrm{U} / \mathrm{ml})$ before retroperfusion, the pancreatic bicarbonate curve appeared to have been shifted to an intermediate position (Fig. 5). Retroperfusion with heparin (500 $\mathrm{U} / \mathrm{ml}$ ) in saline did not alter the normal pancreatic bicarbonate curve.

Retroperfusion of the pancreatic ductal system with a $10^{-6} \mathrm{M}$ solution of the poly-cation poly-L-lysine resulted in a total loss of the normal pancreatic bicarbonate curve (Fig. 6). The bicarbonate concentration of the juice remained constant at a value of approximately $100 \mathrm{meq} / \mathrm{liter}$ irrespective of the flow rate.

Effect of retroperfusion on bicarbonate output. For each of the experimental conditions, the bicarbonate output $30 \mathrm{~min}$ and 60 min after secretin stimulation was calculated. The results are presented in Table 2. The bicarbonate output of the reserpinized animals was significantly less than that of the controls both 30 $\min (P<0.005)$ and $60 \mathrm{~min}(P<0.010)$ after secretin stimulation. Retroperfusion with any of the test substances did not alter the bicarbonate output in the first $30 \mathrm{~min}$ after secretin stimulation. Sixty minutes after secretin, the bicarbonate output was still not altered if the pancreas was first retroperfused with either control saliva, control pancreatic juice or juice obtained from reserpinized rabbits, which was mixed with heparin. By contrast, the bicarbonated output 60 min after secretin stimulation was significantly elevated over that of controls if the pancreatic ductal system was retroperfused with either pancreatic juice collected from reserpinized animals $(P<0.05)$ or poly-L-lysine $(P<0.05)$. Retroperfusion with $C \dot{F}$ saliva also elevated the bicarbonate output, but this fell just short of being significant.

\section{DISCUSSION}

This study demonstrates that reserpinized rabbits secrete pancreatic juice containing a "transport inhibitory factor" that pre-

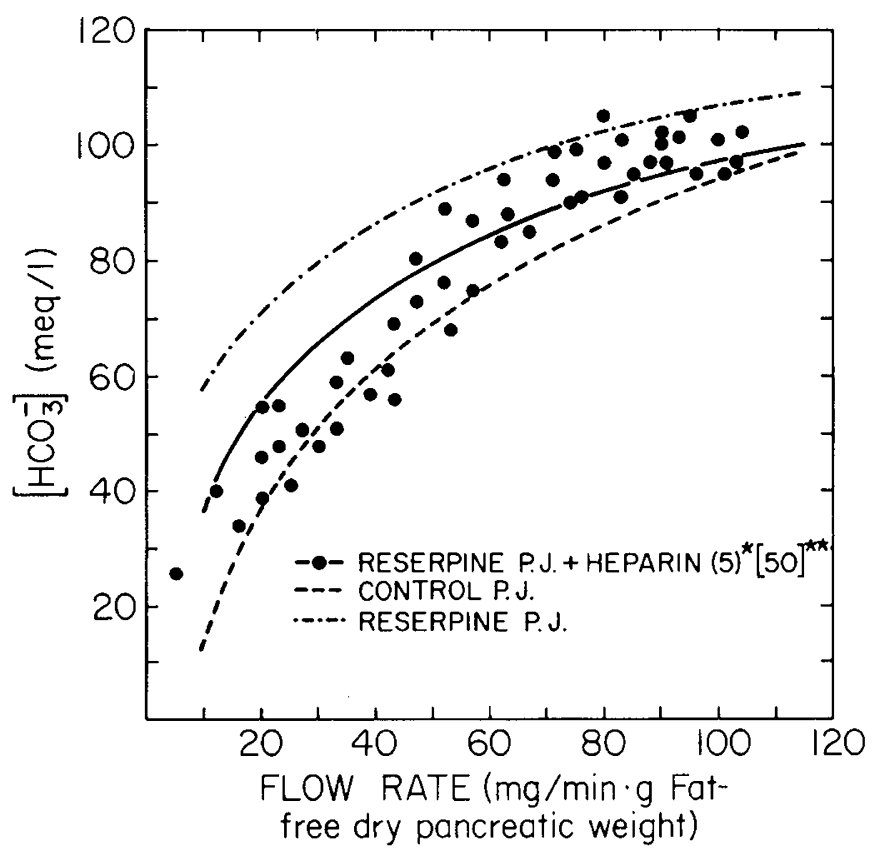

Fig. 5. Bicarbonate concentration as a function of the pancreatic juice flow rate after retroperfusion with a mixture of reserpinized pancreatic juice and heparin. Each point represents a single pancreatic juice sample. The solid line represents the best equation fit for the data points. The dotted lines are the control pancreatic juice curve and the reserpinized pancreatic juice curve from Figure 4. ${ }^{*}$ Number in parentheses represents the number of animals used in the study. ${ }^{* *}$ Number in brackets represents the total number of sample determinations.

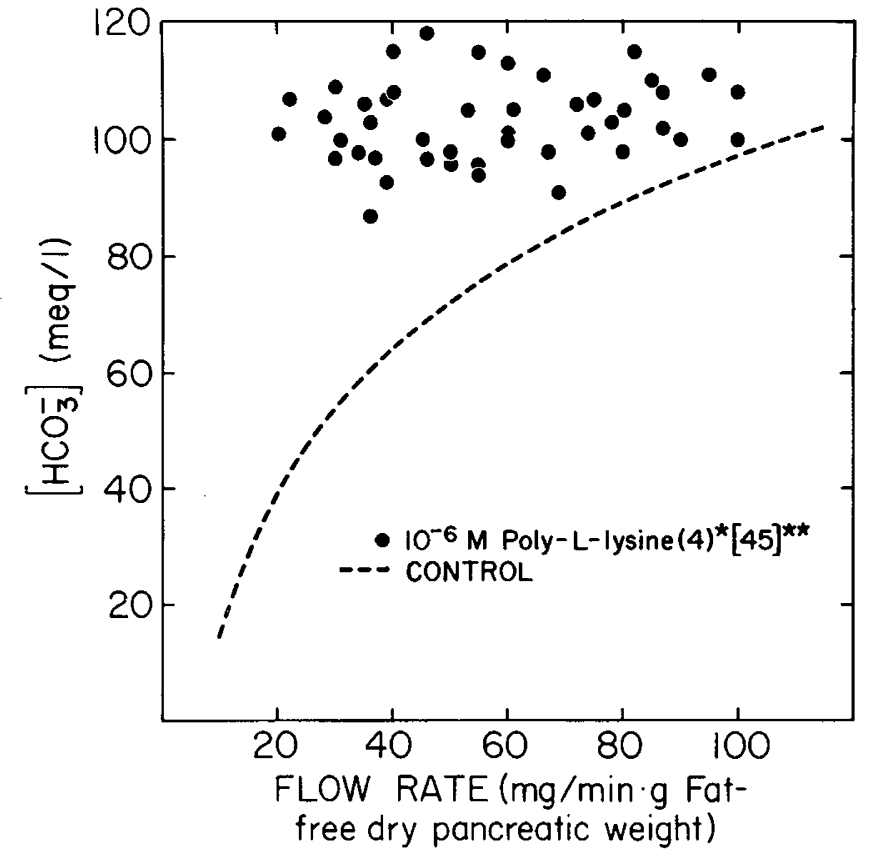

Fig. 6. Bicarbonate concentration as a function of the pancreatic juice flow rate after retroperfusion with poly-L-lysine. Each point represents a single pancreatic juice sample. The dotted line is the control curve from Figure 2. * Number in parentheses represents the number of animals used in the study. ** Number of parentheses represents the total number of sample determinations.

Table 2. Bicarbonate output $30 \mathrm{~min}$ and $60 \mathrm{~min}$ after secretin stimulation (values are given as $m E q \pm S . E . M$.

\begin{tabular}{lcc}
\hline & $30 \mathrm{~min}$ & $60 \mathrm{~min}$ \\
\hline Control & $91.7 \pm 14.4(6)^{1}$ & $137.4 \pm 19.3(6)$ \\
Reserpine treated & $55.8 \pm 10.4^{2}(5)$ & $91.6 \pm 19.0^{3}(5)$ \\
After retroperfusion with: & & \\
$\quad$ CF saliva & $96.6 \pm 10.9(6)$ & $157.9 \pm 16.8(6)$ \\
$\quad$ Control saliva & $91.2 \pm 11.6(5)$ & $143.1 \pm 19.2(5)$ \\
$\quad$ Pancreatic juice from re- & $93.3 \pm 13.3(6)$ & $162.8 \pm 17.4^{4}(5)$ \\
$\quad$ serpinized animals & & \\
Pancreatic juice from con- & $81.7 \pm 8.6(4)$ & $120.0 \pm 23.7(4)$ \\
$\quad$ trol animals & & \\
Pancreatic juice from re- & $91.1 \pm 13.0(5)$ & $135.2 \pm 14.7(4)$ \\
$\quad$ serpinized animals plus & & \\
$\quad$ heparin & & \\
Poly-L-lysine & $98.2 \pm 12.6(4)$ & $170.6 \pm 18.9^{4}(4)$ \\
\hline
\end{tabular}

${ }^{1}$ Number in parenthesis indicates number of animals.

${ }^{2}$ Statistically significant from control; $P<0.005$.

${ }^{3}$ Statistically significant from control; $P<0.010$

${ }^{4}$ Statistically significant from control; $P<0.05$.

vents the normal reabsorption of bicarbonate. To demonstrate this, pancreatic juice obtained from reserpine-treated rabbits was retroperfused into the pancreatic ductal system. The result was a leftward shift in the pancreatic bicarbonate curve (Fig. 4). Retroperfusion with saliva obtained from patients with $\mathrm{CF}$, a solution known to contain a transport inhibitory factor, resulted in a similar shift of the bicarbonate curve (Fig. 3). Retroperfusion with these substances also resulted in an increase in the bicarbonate output (Table 2). This is the result that would be expected if indeed this factor was altering ductal bicarbonate reabsorption. The pancreatic factor was demonstrated to be cationic because its transport inhibitory properties were lost when it was mixed with the polyanion heparin before retroperfusion (Fig. 5). In addition, retroperfusion with the polycation poly-L-lysine inhibited bicarbonate 
reabsorption at all flow rates (Fig. 6), a result which has been observed previously (9).

A second abnormality observed in this study is that the pancreatic juice secreted by reserpine-treated rabbits contained an elevated bicarbonate concentration (Fig. 2). We believe this is due to the transport inhibitory factor. It is important to note, however, that despite the presence of the factor, the total bicarbonate output in the juice of reserpinized rabbits was significantly less than that observed for controls (Table 2). This observation confirms previous studies made in reserpinized rabbits (17) and rats (14). By contrast, the bicarbonate ouput after retroperfusion with substances containing known transport inhibitory properties was elevated over that of controls (Table 2); thus, the reduced bicarbonate output must have been secondary to the reduced volume of pancreatic juice secreted by the reserpinized animals (Table 1) $(14,16,17)$. This decreased volume of juice could be due to obstruction of the pancreatic ductal system. Recent evidence, however, indicates that this is probably the result of a decreased water content in the pancreatic secretions of reserpinized animals (18).

In patients with $\mathrm{CF}$, ductal modification of sweat and saliva is altered by the presence of a transport inhibitory factor $(4,6,7)$. By nature, such a factor would be expected to perturb the movement of reabsorbed ions. In the salivary glands, the major ion reabsorbed is sodium (10); therefore, this factor inhibits sodium movement (8). The sweat gland ductal system reabsorbs both sodium and bicarbonate (5). In patients with $\mathrm{CF}$, these glands secrete a fluid which contains an increased concentration of sodium (4) and bicarbonate (5); again, this is the result of the transport inhibitory factor. In a more recent study, bicarbonate concentration of CF sweat was observed to be reduced because of inhibition of a newly described chloride/bicarbonate exchange mechanism (15). This inhibition could very well be secondary to the transport inhibitory factor blocking chloride reabsorption. In the pancreas, the ion that is reabsorbed is bicarbonate (19). If a transport inhibitory factor is also present in this gland, one would expect the pancreatic juice concentration of this anion to be elevated. This was observed in this study.

During the past few years, many studies have demonstrated that reserpine-treated animals produce glandular secretions that are similar to those produced by persons with $\mathrm{CF}$. The saliva of reserpinized rats contains an elevated sodium concentration (12). This is due to a positively charged transport inhibitory factor (13). The pancreas of reserpine-treated rats and rabbits secrete reduced amounts of juice which contains elevated amounts of protein but a decreased bicarbonate output $(14,17)$. In this study, the pancreatic juice secreted by treated rabbits was also shown to contain a decreased bicarbonate output (Table 1) but an elevated bicarbonate concentration (Fig. 2). It is believed that this latter finding is due to a "transport inhibitory factor" that blocks the reabsorption of bicarbonate. Whether or not a similar factor is also present in patients with CF has not yet been elucidated. One recent study of pancreatic function, however, has indicated that patients with CF have defective bicarbonate secretion at all levels of pancreatic enzyme secretion (1). Although this observation in itself does not directly support our findings, it does point out the complexity of this problem. Clearly, more extensive studies are needed to fully investigate this finding and to establish the existence of a transport inhibitory factor in the pancreatic secretions of patients with $\mathrm{CF}$.

\section{REFERENCES AND NOTES}

1. Gaskin, K. and Forstner, G.: Evidence for a primary defect of pancreatic $\mathrm{HCO}_{3}{ }^{-}$ secretion in cystic fibrosis. Pediatr. Res. 16: 554 (1982).

2. Hadorn, B., Johansen, P. G., and Anderson, C. M.: Pancreozymin secretin test of exocrine pancreatic function in cystic fibrosis and the significance of the result for the pathogenesis of the disease. Can. Med. Assn. J., 98: 377 (1968).

3. Immken, L., Martinez, J. R., Hess, R. A., and Barbero, G. J.: The chronically reserpinized rat as a possible model for cystic fibrosis. III. Histochemistry of exocrine glands. Cystic Foundation Club Abstracts, XVI Annual Meeting (1975).

4. Kaiser, D., Drack, E., and Rossi, E.: Inhibition of net sodium transport in single sweat glands by sweat of patients with cystic fibrosis of the pancreas. Pediatr. Res. 5: 167 (1971).

5. Kaiser, D. and Drack, E.: Diminished excretion of bicarbonate from the single sweat gland of patients with cystic fibrosis of the pancreas. Europ. J. Clin. Invest., 4: 261 (1974).

6. Mangos, J. A. and McSherry, N. R.: Sodium transport: Inhibitory factor in sweat of patients with cystic fibrosis. Science, 158: 135 (1967).

7. Mangos, J. A., McSherry, N. R., and Benke, P. J.: A sodium transport inhibitory factor in the saliva of patients with cystic fibrosis of the pancreas. Pediatr. Res., 1: 436 (1967).

8. Mangos, J. A. and McSherry, N. R.: Studies on the mechanism of inhibition of sodium transport in cystic fibrosis of the pancreas. Pediatr. Res., 2: 378 (1968).

9. Mangos, J. A. and McSherry, N. R.: Micropuncture study of excretion of water and electrolytes by the pancreas. Am. J. Physiol., 22: 1496 (1971).

10. Martinez, J. R., Holzgreve, H., and Frick, A.: Micropuncture study of submaxillary glands of adult rats. Pflugers Arch., 290: 124 (1966).

11. Martinez, J. R., Adelstein, E., Quissel, D., and Barbero, G. J.: The chronically reserpinized rat as a possible model for cystic fibrosis. I. Submaxillary gland morphology and ultrastructure. Pediatr. Res. 9: 463 (1975).

12. Martinez, J. R., Adshead, P. C., Quissel, D. O., and Barbero, G. J.: The chronically reserpinized rat as a possible model for cystic fibrosis. II. Composition and cilioinhibitory effects of submaxillary saliva. Pediatr. Res., 9: 470 (1975).

13. Martinez, J. R., Martinez, A. M., Garrett, L., and Korman, P.: The chronically reserpinized rat as a model for cystic fibrosis: $\mathrm{Na}^{+}$transport inhibitory effect in submaxillary saliva. Pediatr. Res., 13: 1156 (1979).

14. Perlmutter, J. and Martinez, J. R.: The chronically reserpinized rat as a possible model for cystic fibrosis. VII. Alterations in the secretory response to cholecystokinin and to secretin from the pancreas in vivo. Pediatr. Res., 12: 188 (1978).

15. Quinton, P. M.: Suggestion of an abnormal anion exchange mechanism in sweat of cystic fibrosis patients. Pediatr. Res., 16: 533 (1982).

16. Shiffman, M. L., Gillon, M. J., and Galey, W. R.: Water and electrolyte secretion by the pancreas of the reserpinized rabbit: A model for cystic fibrosis. In: J. M. Sturges, Ed. Perspectives in Cystic Fibrosis: Proceedings of the Eighth International Congress on Cystic Fibrosis. pp. 92-97. Cystic Fibrosis Foundation, Toronto, Canada, 1980.

17. Shiffman, M. L., Gillon, M. J., and Galey, W. R.: Pancreatic function in the reserpinized rabbit-a model for cystic fibrosis. I. Effect of secretin. Pediatr. Res., 16: 104 (1982).

18. Shiffman, M. L., Gillon, M. J., and Galey, W. R.: A barrier to water movement in the pancreas of reserpine treated rabbits-a model for cystic fibrosis. Pediatr. Res., submitted for publication, 1983

19. Swanson, C. H. and Solomon, A. K.: Micropuncture analysis of the cellular mechanisms of electrolyte secretion by the in vitro rabbit pancreas. J. Gen. Physiol., 65: 22 (1975).

20. Wood, R. E., Boat, T. F., and Doershuk, C. F.: State of the art: cystic fibrosis. Am. Rev. Resp. Dis., 113: 833 (1976).

21. Zoppi, G., Shmerling, D. H., Gaburro, D., and Prader, A.: The electrolyte and protein contents and outputs in duodenal juice after pancredzymin and secretin stimulation in normal children and in patients with cystic fibrosis. Acta Paediatr. Scand., 59: 692 (1970).

22. The authors would like to acknowledge the assistance of Mary Lou Farnett for her work on this manuscript.

23. Recipient of a Cystic Fibrosis Foundation Student Traineeship during this study. 24. Supported by grants from the NIH, grant \#2RO1 AM17843 and the Hendricks Foundation of the SUNY Research Foundation, grant \#2RO7 RR0540220.

25. Address repint requests to: Dr. Phillip T. Swender, Robert C. Schwartz Cystic Fibrosis Center, Dept. of Pediatrics, SUNY, Upstate Medical Center, Syracuse, NY 13210.

26. Received for publication May 12, 1982.

27. Accepted for publication January 13, 1983. 